Research Paper

\title{
Elevated Serum S100A9 Indicated Poor Prognosis in Hepatocellular Carcinoma after Curative Resection
}

\author{
Jun Meng ${ }^{1^{*}}$, Feng $\mathrm{Gu}^{2^{*}}$, Hua Fang${ }^{2}$, Bin $\mathrm{Qu}^{1{ }^{凶}}$ \\ 1. Department of Clinical Laboratory, Ruijin Hospital, Shanghai Jiao Tong University School of Medicine, No. 197, Rui Jin Er Road, Shanghai 200025, P.R. \\ China \\ 2. Department of Clinical Laboratory, People's Hospital of Shanghai Pudong District, No. 490, Chuan Huan Road South, Chuansha Town, Shanghai 201200, \\ P.R. China \\ * These authors contributed equally to this work. \\ $\square$ Corresponding author: Bin Qu, BSM, Department of Clinical Laboratory, Ruijin Hospital, Shanghai Jiao Tong University School of Medicine, No. 197, Rui Jin \\ Er Road, Shanghai 200025, P.R. China. Tel. \& Fax: +86-21-64370045. E-mail: qb3793@163.com. \\ (c) Ivyspring International Publisher. This is an open access article distributed under the terms of the Creative Commons Attribution (CC BY-NC) license \\ (https://creativecommons.org/licenses/by-nc/4.0/). See http://ivyspring.com/terms for full terms and conditions.
}

Received: 2018.07.09; Accepted: 2018.10.24; Published: 2019.01.01

\begin{abstract}
Background: Previous studies suggest S100A9 is a promising biomarker for prognosis in cancer, including hepatocellular carcinoma (HCC). We examined the utility of serum S100A9 in predicting prognosis in $\mathrm{HCC}$ after curative resection.

Methods: We conducted a retrospective study of $379 \mathrm{HCC}$ patients who underwent curative resection. Patients were randomly stratified into two independent groups to evaluate the prognostic value of S100A9. S100A9 was determined by ELISA.

Results: Patients with advanced disease showed significantly higher S100A9 levels (all $P<0.050$ ). Serum S100A9 was elevated in patients who developed recurrence and death in both training and validation cohorts (all $P<0.050$ ). In the training cohort, patients with higher preoperative S100A9 had a significantly shorter time to recurrence $(15.50$ vs. 64.00 months, $P<0.001)$ and decreased overall survival ( 34.80 months vs. not reached, $P<0.001$ ). Cox regression demonstrated S100A9 was an independent indicator for poor prognosis after resection (both $P<0.050$ ). These results were confirmed by the independent validation cohort.

Conclusions: Serum S100A9 is associated with dismal outcomes in HCC patients and can serve as a novel prognostic indicator for $\mathrm{HCC}$ patients after resection. Determination of S100A9 might help tailor treatment strategy to improve HCC patient prognosis.
\end{abstract}

Key words: hepatocellular carcinoma, serum biomarker, S100A9, prognosis, curative resection

\section{Introduction}

Hepatocellular carcinoma (HCC) is the fifth most prevalent malignant disease and the third leading cause of cancer-related death worldwide [1-3]. Surgical resection is currently considered as the only approach with curative potential for HCC patients [4, 5]. However, the prognosis of HCC patients after curative resection remains unsatisfactory due to high recurrence and metastasis incidence [6-8]. Thus, there is an urgent need for a reliable biomarker that can effectively detect patients with a poor prognosis so that additional treatments can be administered.
S100A9 is a calcium-binding protein that forms homodimers or heterodimers with S100A8 to play a vital role in inflammatory processes [9-11]. Previous studies have demonstrated that S100A9 is upregulated in several kinds of solid tumors, including colorectal [12], prostate [13], and breast cancer [14]. Importantly, overexpression of S100A9 has been found to be positively correlated with poor differentiation, tumor aggressiveness, metastasis, and worse clinical outcomes in these cancers [15-17], which has indicated its critical significance in 
mediating tumor progression. These findings suggest that S100A9 could serve as a promising biomarker for prognosis evaluation in cancer.

Previous investigations indicated that S100A9 was overexpressed in HCC tissues and could promote HCC cell growth and invasion through RAGEdependent MAPK signaling cascades [18-20]. More importantly, systemic quantitative proteomics analysis showed that serum concentrations of S100A9, a secreted protein, were significantly upregulated in HCC patients, and these elevated levels showed satisfactory diagnostic potentials [21]. However, whether elevated serum S100A9 shows prognostic value has not been examined.

In light of the above findings, we designed a retrospective, single-center study with an independent validation cohort to investigate the prognostic potential of serum S100A9 in HCC patients receiving curative resection. We found that patients that encountered dismal outcomes showed significantly higher serum S100A9 levels. Our results indicate that serum S100A9 could serve as a novel, reliable, and promising circulating biomarker for prognosis evaluation in HCC.

\section{Patients and Methods}

\section{Study population}

We retrospectively recruited 379 HCC patients who underwent curative resection at Ruijin Hospital (Jiaotong University, Shanghai, China) between January and December 2012. Patients were randomized into the training cohort $(n=189)$ and validation cohort $(n=190)$ (Figure 1). HCC was defined based on imaging scans and biochemistry tests and was confirmed by histopathology according to the American Association for the Study of Liver Diseases guidelines [22]. The HCC tumor stage was defined according to the Barcelona Clinic Liver Cancer (BCLC) staging system, and BCLC 0+A stage tumors were classified as early stage [23]. Tumor differentiation was determined according to the Edmondson grading system. Liver function was assessed by the Child-Pugh scoring system. Approval for the use of human subjects was obtained from the research ethics committee of Ruijin Hospital, and informed consent was obtained from each individual enrolled in the study.

\section{Follow up}

Follow up was conducted according to the procedures of a previous study ${ }^{24}$. Briefly, patients were followed up every month during the first 6 months post-treatment and every 3 to 4 months thereafter. All patients were monitored by abdominal ultrasonography and chest X-ray every 1 to 6 months and by computed tomography scans every 6 months. Bone scans or magnetic resonance imaging scans were performed if localized bone pain was reported. Follow up ended in December 2017. Time to recurrence (TTR) was defined as the interval between surgery and the diagnosis of any type of recurrence, including intrahepatic or extrahepatic recurrence as identified by magnetic resonance imaging or computed tomography [24]. Overall survival (OS) was defined as the interval between treatment and death of any cause (months) or the last observation date [24].

\section{Determination of serum S100A9 levels}

Pre-treatment serum $(3 \mathrm{ml})$ was collected within 2 days before treatment for S100A9 concentration determination. Serum S100A9 was determined by enzyme-linked immunosorbent assay (ELISA) using the Human S100A9 ELISA Kit (Cusabio Biotech) according to the manufacturer's instructions. The detection range of the ELISA kit was 4.69-300 ng/ml, and serum samples beyond this range were further diluted before performing ELISA tests. The cutoff value of serum S100A9 level for diagnosis and prognosis was set at $252.40 \mathrm{ng} / \mathrm{ml}$, according to the median value in the training cohort.

\section{Statistical analysis}

Statistical analyses were performed using SPSS 20.0 software (IBM, Chicago, USA). Experimental values are presented as the mean \pm SEM for continuous variables. $\chi^{2}$ tests, Fisher's exact probability tests and Student's $t$-tests were used for comparison between groups, as appropriate. If variances within groups were not homogeneous, the nonparametric Mann-Whitney $U$ test or Wilcoxon signed-rank test was used. The prognostic value of S100A9 was evaluated by Kaplan-Meier survival curves, log-rank tests, and Cox proportional hazards models. A p-value less than 0.05 was considered significant.

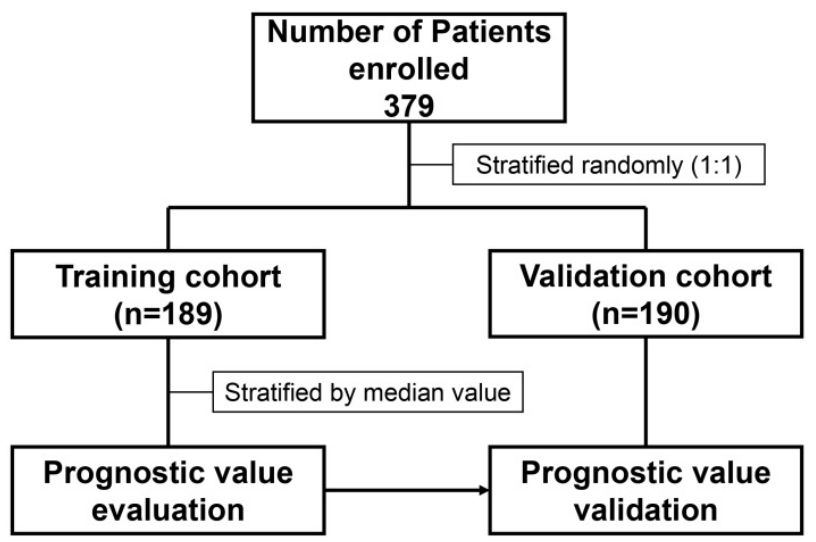

Figure 1. Distribution of patients enrolled in present study. 


\section{Results}

\section{Patient characteristics}

The clinicopathologic characteristics of patients are shown in Table 1. The mean patient age was 54.78 years (range: $25-82$ years) in the training cohort, and 52.41 years ( $29-75$ years) in the validation cohort. The major cause of liver disease was hepatitis B virus (HBV). All of the tumor characteristics except alphafetoprotein (AFP, $\mathbf{P = 0 . 0 3 3 )}$ were similar between the training and validation cohorts. During the follow-up period, $68.78 \%$ of patients $(130 / 189)$ in the training cohort encountered recurrence, while $67.89 \%$ of patients $(129 / 190)$ in the validation cohort encountered recurrence. Almost half $(48.15 \%, 91 / 189)$ of the patients in the training cohort died during follow-up, and a similar rate of death was observed in the validation cohort $(51.05 \%, 97 / 190)$.

Table 1. Patient characteristics in training and validation cohorts

\begin{tabular}{|c|c|c|c|c|c|c|c|}
\hline \multirow{2}{*}{\multicolumn{2}{|c|}{ Characteristic }} & \multirow{2}{*}{$\begin{array}{l}\text { No. of } \\
\text { patients }\end{array}$} & \multicolumn{2}{|c|}{ Training cohort } & \multicolumn{2}{|c|}{ Validation cohort } & \multirow[t]{2}{*}{$P$} \\
\hline & & & No. & $\%$ & No. & $\%$ & \\
\hline \multicolumn{2}{|c|}{ Total No. of patients } & 379 & 189 & & 190 & & \\
\hline \multirow[t]{2}{*}{ Sex } & Male & 323 & 167 & 88.36 & 156 & 82.11 & 0.086 \\
\hline & Female & 56 & 22 & 11.64 & 34 & 17.89 & \\
\hline \multirow[t]{2}{*}{ Age } & $\leq 50$ & 123 & 59 & 31.21 & 64 & 33.68 & 0.608 \\
\hline & $>50$ & 256 & 130 & 68.79 & 126 & 66.32 & \\
\hline \multirow[t]{2}{*}{$\operatorname{AFP}(\mathrm{ng} / \mathrm{mL})$} & $\leq 400$ & 249 & 134 & 70.90 & 115 & 60.53 & 0.033 \\
\hline & $>400$ & 130 & 55 & 29.10 & 75 & 39.47 & \\
\hline \multirow[t]{2}{*}{$\operatorname{ALT}(\mathrm{U} / \mathrm{L})$} & $\leq 75$ & 333 & 171 & 90.48 & 162 & 85.26 & 0.120 \\
\hline & $>75$ & 46 & 18 & 9.52 & 28 & 14.74 & \\
\hline \multirow{2}{*}{$\begin{array}{l}\text { Liver } \\
\text { cirrhosis }\end{array}$} & No & 64 & 37 & 19.58 & 27 & 14.21 & 0.163 \\
\hline & Yes & 315 & 152 & 80.42 & 163 & 85.79 & \\
\hline \multirow[t]{2}{*}{ No.of tumor } & Single & 301 & 146 & 77.25 & 155 & 81.58 & 0.297 \\
\hline & Multiple & 78 & 43 & 22.75 & 35 & 18.42 & \\
\hline \multirow{2}{*}{$\begin{array}{l}\text { Tumor size, } \\
\mathrm{cm}\end{array}$} & $\leq 5$ & 230 & 111 & 58.73 & 119 & 62.63 & 0.437 \\
\hline & $>5$ & 149 & 78 & 41.27 & 71 & 37.37 & \\
\hline \multirow{2}{*}{$\begin{array}{l}\text { Vascular } \\
\text { invasion }\end{array}$} & No & 234 & 119 & 62.96 & 115 & 60.53 & 0.626 \\
\hline & Yes & 145 & 70 & 37.04 & 75 & 39.47 & \\
\hline \multirow{2}{*}{$\begin{array}{l}\text { Edmondson } \\
\text { stage }\end{array}$} & I-II & 229 & 115 & 60.85 & 114 & 60.00 & 0.866 \\
\hline & III-IV & 150 & 74 & 39.15 & 76 & 40.00 & \\
\hline \multirow{2}{*}{$\begin{array}{l}\text { Child-Pugh } \\
\text { score }\end{array}$} & A & 364 & 184 & 97.35 & 180 & 94.74 & 0.191 \\
\hline & B & 15 & 5 & 2.65 & 10 & 5.26 & \\
\hline \multirow[t]{2}{*}{ BCLC stage } & $0+A$ & 312 & 157 & 83.07 & 155 & 81.58 & 0.704 \\
\hline & $B+C$ & 87 & 32 & 16.93 & 35 & 18.42 & \\
\hline
\end{tabular}

Abbreviations: AFP, a-fetoprotein; ALT, alanine aminotransferase; $\mathrm{HBsAg}$, hepatitis B surface antigen; BCLC, Barcelona Clinic Liver Cancer

\section{Patient with advanced disease exhibited higher serum S100A9}

In the training cohort, serum S100A9 concentrations were significantly higher in patients with advanced tumor stage (termed as BCLC stage B+C, $381.7 \pm 41.68$ vs. $257.50 \pm 11.76, P<0.001$, Figure $2 \mathrm{~A}$ ), poor differentiation $(308.90 \pm 19.86$ vs. $259.00 \pm 15.82$, $P=0.044$, Figure 2B), multiple lesions $(336.00 \pm 31.19$ vs. $261.60 \pm 13.00, P=0.012$, Figure $2 C$ ), and vascular invasion $(302.00 \pm 21.88$ vs. $268.80 \pm 15.05, P=0.042$, Figure 2D). Similar results were observed in the validation cohort (all $P<0.050$, Figure 2A-D). Taken together, these findings suggested that serum S100A9 might be a potential indicator for reflecting the severity of HCC.
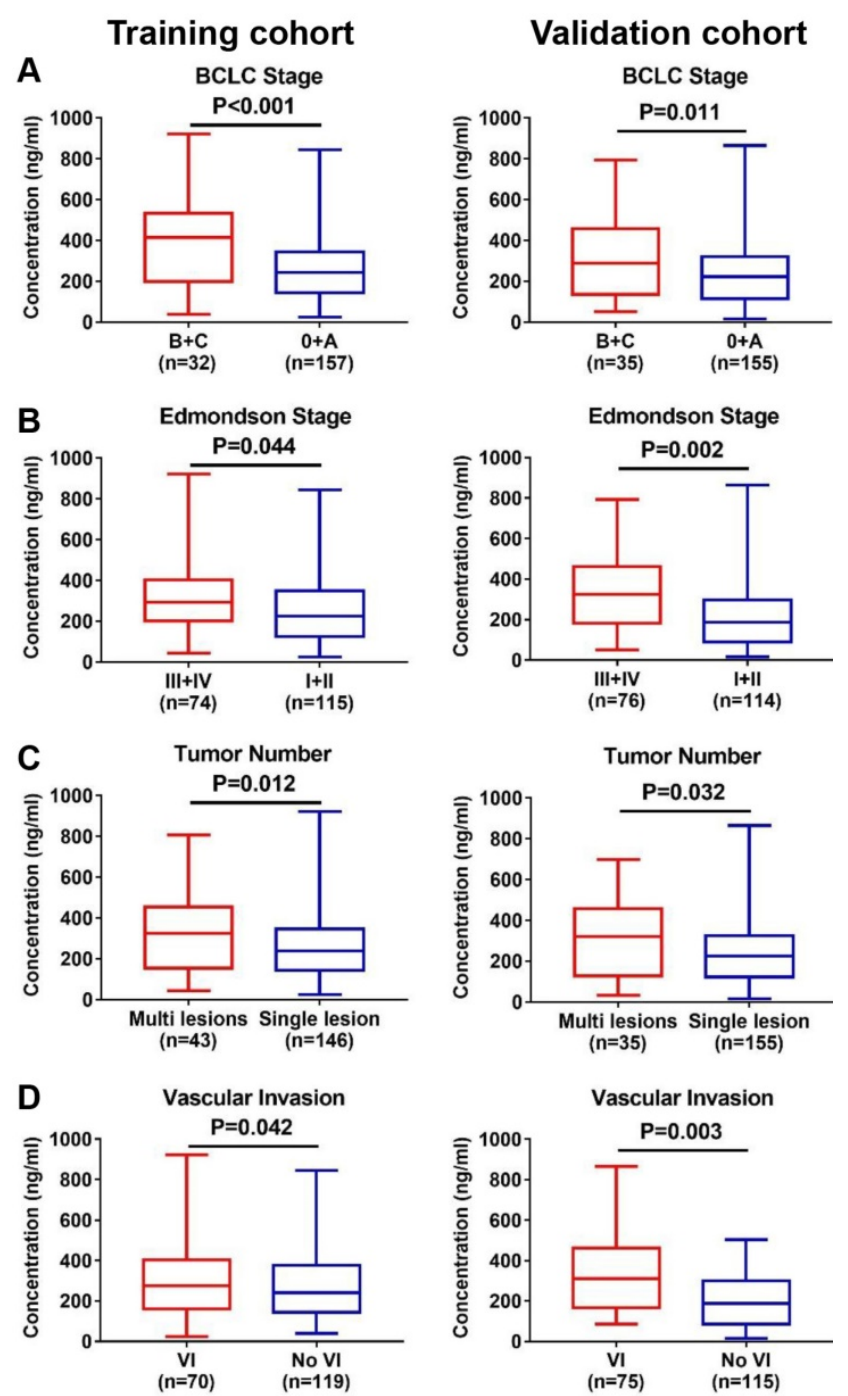

Figure 2. Correlation between serum S100A9 level and severity of HCC. (A) Distribution of serum S100A9 levels in different BCLC stage of training (left) and validation (right) cohort. (B) Distribution of serum S100A9 levels in different Edmondson stage of training (left) and validation (right) cohort. (C) Distribution of serum S100A9 levels in patients with different tumor number of training (left) and validation (right) cohort. (D) Distribution of serum S100A9 levels in patients with or without vascular invasion of training (left) and validation (right) cohort.

\section{Serum S100A9 concentration was significantly elevated in patients who suffered poor outcome}

Next, we explored the correlation between serum S100A9 level and prognosis. In the training cohort, serum S100A9 level was significantly higher in recurrent patients than in non-recurrent patients (336.40 \pm 17.35 vs. $157.10 \pm 16.20, P<0.001$, Figure $3 A$ ). Similarly, S100A9 level was significantly higher in patients who died during follow up than in those who were alive at the end of the study $(333.30 \pm 14.75$ vs. 227.70 $\pm 18.39, P<0.001$, Figure 3B). 

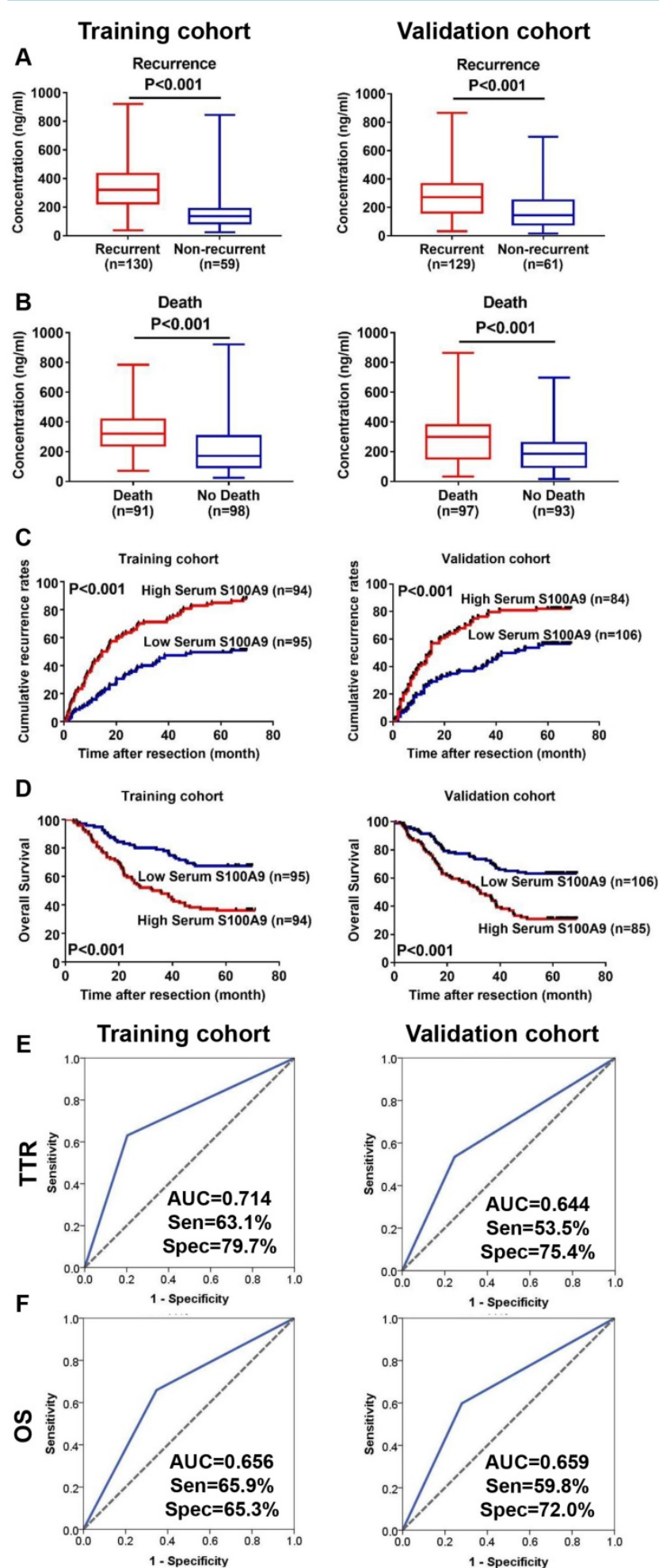

Figure 3. Serum S100A9 as an indicator for HCC prognosis after curative resection. (A) Distribution of serum S100A9 levels in patient with different recurrence status of training (left) and validation (right) cohort. (B) Distribution of serum S100A9 levels in patient with different survival status of training (left) and validation (right) cohort. (C) Kaplan-Meier analysis of HCC patients according to serum S100A9 levels in training (left) and validation (right) cohort for predicting recurrence. (D) Kaplan-Meier analysis of HCC patients according to serum S100A9 levels in training (left) and validation (right) cohort for predicting overall survival. $(E)$ ROC analysis of HCC patients according to serum S100A9 levels in training (left) and validation (right) cohort for predicting recurrence. (F) ROC analysis of HCC patients according to serum S100A9 levels in training (left) and validation (right) cohort for predicting time to overall survival.
In the validation cohort, we also observed significantly higher S100A9 levels in patients who suffered recurrence $(288.80 \pm 14.82$ vs. $182.50 \pm 17.34, P$ $<0.001$, Figure 3A) and death $(299.00 \pm 17.66$ vs. 204.30 $\pm 14.47, P<0.001$, Figure 3B). These findings revealed that serum S100A9 could be used for predicting HCC prognosis.

\section{Serum S100A9 served as a prognostic indicator in the training cohort}

We first investigated the prognostic significance of serum S100A9 in patients receiving curative resection in the training cohort. During the follow up, $68.78 \%$ of patients $(130 / 189)$ encountered recurrence, and $48.15 \%(91 / 189)$ of patients died. Because there is no widely accepted cutoff value of serum S100A9 for predicting prognosis in HCC, we set the optimal cutoff value as $252.40 \mathrm{ng} / \mathrm{ml}$, according to the median value in the training cohort, and stratified patients into two groups based on S100A9 level. We found that patients with higher serum S100A9 level had significantly shorter TTR (median 15.50 months vs. 64.00, $P<0.001$, Figure $3 \mathrm{C}$ ) and OS (median 34.80 months vs. not reached, $P<0.001$, Figure 3D). Furthermore, ROC analysis were conducted to explore the performances of serum S100A9 for predicting prognosis in training cohort. Results showed that, in training cohort, AUC-ROC of S100A9 for predicting TTR and OS were 0.714 (Sensitivity $63.1 \%$, Specificity $79.7 \%$ ) and 0.656 (Sensitivity $65.9 \%$, Specificity 65.3\%), respectively (Figure 3E, F). While in validation cohort, AUC-ROC of S100A9 for predicting TTR and OS were 0.644 (Sensitivity 53.5\%, Specificity 75.4\%) and 0.659 (Sensitivity 59.8\%, Specificity $72.0 \%$ ), respectively (Figure $3 \mathrm{E}, \mathrm{F}$ ). The multivariate Cox regression analysis revealed that high serum S100A9 level before resection was a significant indicator for both TTR [hazard ratio (HR): 2.315; 95\% confidential interval (95\% CI): 1.409-3.104; $P<0.001$; Table 2] and OS (HR: 2.081; 95\% CI: 1.325-3.270; $P<0.001$, Table 2).

\section{Predicting prognosis of HCC patients with serum S100A9 level in subgroups of the training cohort}

The prognostic significance of serum S100A9 level within low recurrence/death risk subgroups was next investigated. Among patients with AFP $\leq$ $400 \mathrm{ng} / \mathrm{ml}$ (low-AFP), high serum S100A9 level indicated a higher probability of recurrence (median 17.45 months vs. not reached; $P<0.001$; Figure 4A) or death (median 39.21 months vs. not reached; $P<$ 0.001; Figure 4A). In the early-HCC subgroup, higher preoperative serum S100A9 level also indicated a significantly shorter TTR (median 19.77 months vs. 
not reached; $P<0.001)$ or OS $(38.50$ months vs. not reached; $P<0.001$; Figure 4B). Additionally, serum S100A9 retained its prognostic value in patients without vascular invasion (TTR: median 17.40 months vs. not reached; $P<0.001$; OS: 36.00 months vs. not reached; $P<0.001$; Figure 4C) or small tumor (TTR: median 21.05 months vs not reached; $P<0.001$; OS: 51.63 months vs. not reached; $P<0.001$; Figure $4 \mathrm{D}$ ).

Table 2. Multivariate Cox proportional hazard regression analysis of factors associate with prognosis in training and validation cohort

\begin{tabular}{|c|c|c|c|c|c|c|c|}
\hline \multirow{2}{*}{\multicolumn{2}{|c|}{ Characteristic }} & \multicolumn{3}{|c|}{ Training cohort } & \multicolumn{3}{|c|}{ Validation cohort } \\
\hline & & \multirow[t]{2}{*}{ HR } & \multirow[t]{2}{*}{$95 \% \mathrm{CI}$} & \multirow[t]{2}{*}{$\mathbf{P}$} & \multirow[t]{2}{*}{ HR } & \multirow[t]{2}{*}{$95 \% \mathrm{CI}$} & \multirow[t]{2}{*}{$\mathbf{P}$} \\
\hline Indicators fo & r TTR & & & & & & \\
\hline $\begin{array}{l}\mathrm{AFP} \\
(\mathrm{ng} / \mathrm{mL})\end{array}$ & $\begin{array}{l}>400 \text { vs } \\
\leq 400\end{array}$ & 1.644 & $1.110-2.435$ & 0.013 & 1.235 & $0.856-1.781$ & 0.280 \\
\hline No.of tumor & $\begin{array}{l}\text { Multiple vs } \\
\text { Single }\end{array}$ & 1.614 & $1.039-2.506$ & 0.033 & 1.998 & $1.319-3.027$ & 0.001 \\
\hline $\begin{array}{l}\text { Tumor size } \\
(\mathrm{cm})\end{array}$ & $\begin{array}{l}>5 \text { vs } \\
\leq 5\end{array}$ & 2.039 & $1.429-3.066$ & $<0.001$ & 2.89 & $1.528-4.485$ & $<0.001$ \\
\hline $\begin{array}{l}\text { Vascular } \\
\text { invasion }\end{array}$ & $\begin{array}{l}\text { Yse vs } \\
\text { No }\end{array}$ & 1.226 & $0.840-1.788$ & 0.290 & N.A. & & \\
\hline $\begin{array}{l}\text { Edmondson } \\
\text { stage }\end{array}$ & $\begin{array}{l}\text { III-IV vs } \\
\text { I-II }\end{array}$ & 1.070 & $0.734-1.539$ & 0.726 & 1.334 & 0.904-1.967 & 0.146 \\
\hline $\begin{array}{l}\text { Child-Puge } \\
\text { stage }\end{array}$ & $\begin{array}{l}\text { B vs } \\
\text { A }\end{array}$ & 1.908 & $0.744-4.895$ & 0.179 & N.A. & & \\
\hline $\begin{array}{l}\text { S100A9 } \\
\text { (ng/ml) }\end{array}$ & $\begin{array}{l}\leq 242.5 \text { vs } \\
>242.5\end{array}$ & 2.315 & $1.409-3.104$ & $<0.001$ & 1.833 & $1.241-2.707$ & 0.002 \\
\hline \multicolumn{8}{|c|}{ Indicators for OS } \\
\hline $\begin{array}{l}\text { AFP } \\
(\mathrm{ng} / \mathrm{mL})\end{array}$ & $\begin{array}{l}>400 \text { vs } \\
\leq 400\end{array}$ & 1.659 & $1.060-2.599$ & 0.027 & N.A. & & \\
\hline No.of tumor & $\begin{array}{l}\text { Multiple vs } \\
\text { Single }\end{array}$ & 1.770 & $1.093-2.867$ & 0.002 & 2.195 & $1.390-3.465$ & 0.001 \\
\hline $\begin{array}{l}\text { Tumor size } \\
(\mathrm{cm})\end{array}$ & $\begin{array}{l}>5 \text { vs } \\
\leq 5\end{array}$ & 2.056 & $1.320-3.202$ & 0.001 & 1.712 & $1.128-2.597$ & $<0.001$ \\
\hline $\begin{array}{l}\text { Vascular } \\
\text { invasion }\end{array}$ & $\begin{array}{l}\text { Yse vs } \\
\text { No }\end{array}$ & 1.164 & $0.712-1.744$ & 0.636 & N.A. & & \\
\hline $\begin{array}{l}\text { Child-Puge } \\
\text { stage }\end{array}$ & $\begin{array}{l}\text { B vs } \\
\text { A }\end{array}$ & 2.413 & $0.840-6.930$ & 0.102 & 1.061 & $0.425-2.650$ & 0.899 \\
\hline $\begin{array}{l}\text { S100A9 } \\
\text { (ng/ml) }\end{array}$ & $\begin{array}{l}\leq 242.5 \text { vs } \\
>242.5\end{array}$ & 2.081 & $1.325-3.270$ & $<0.001$ & 1.948 & $1.250-3.035$ & 0.003 \\
\hline
\end{tabular}

Abbreviations: TTR, time to recurrence; OS, overall survival; AFP, a-fetoprotein; ALT, alanine aminotransferase; BCLC, Barcelona Clinic Liver Cancer

\section{Validation of serum S100A9 level as a prognostic indicator for HCC}

To validate the prognostic value of serum S100A9, an independent cohort containing 190 HCC patients underwent curative resection was recruited. In the validation cohort, the TTR for patients with high serum S100A9 level was significantly shorter compared with those with low S100A9 level (median 14.75 months vs 44.75, $P<0.001$, Figure 3C). Patients with high serum S100A9 level also showed significantly shorter OS (median 32.50 months vs. not reached, $P<0.001$, Figure 3D). Multivariate Cox regression confirmed that high serum S100A9 level was an independent indicator for both TTR (HR 1.833; 95\% 1.241-2.707; $P=0.002$; Table 2) and OS (HR 1.948; 95\% 1.250-3.035; $P=0.003$; Table 2$)$ in the validation cohort.
The prognostic value of serum S100A9 was also validated in low-recurrence/death-risk subgroups, and the results showed that serum S100A9 retained its prognostic value in the low-AFP subgroup (TTR: median 15.00 months vs. $45.33, P=0.009$; OS: 38.75 vs. not reached, $P=0.003$; Figure $4 \mathrm{~A}$ ), early-stage (TTR: median 15.00 months vs. $49.83, P<0.001$; OS: 38.50 vs. not reached, $P<0.001$; Figure 4B), no vascular invasion (TTR: median 14.00 months vs. $42.00, P=0.001$; OS: 34.00 vs. not reached, $P=0.005$; Figure $4 \mathrm{C}$ ), and small tumor size subgroups (TTR: median 16.50 months vs. 51.23, $P=0.002$; OS: 38.50 vs. not reached, $P<0.001$; Figure 4D). Collectively, our data successfully validated the prognostic value of serum S100A9 in HCC.

\section{Correlation between serum S100A9 level and clinical characteristics}

In training cohort, patients with high serum S100 A9 level were prone to have a larger tumor than those with low S100A9 level $(P=0.033$, Table 3$)$. Moreover, patients with high serum S100A9 level were also likely to have multiple tumor lesions, though it did not reach statistical significance $(P=0.051$, Table 3$)$. However, there was no significant difference in other clinicopathological characteristics including pathological differentiation between high and low S100A9 level groups in the training cohort. Meanwhile, in validation cohort, high S100A9 level significantly correlated with multiple tumor numbers $(P=0.014)$, vascular invasion $(P=0.001)$, poor differentiation $(P=$ $0.001)$, and advanced BCLC stage $(P=0.037$, Table 3$)$.

Table 3. Correlation between serum S100A9 and clinicopathological parameters

\begin{tabular}{|c|c|c|c|c|c|c|c|}
\hline \multicolumn{2}{|c|}{ Characteristics } & \multicolumn{3}{|c|}{ Training cohort } & \multicolumn{3}{|c|}{ Validation cohort } \\
\hline Sex & Male & $\begin{array}{l}\text { S100A9 } \\
\leq 242.5 \\
83\end{array}$ & $\begin{array}{l}\text { S100A9 } \\
>242.5 \\
84\end{array}$ & $\begin{array}{l}\boldsymbol{P} \\
0.669\end{array}$ & $\begin{array}{l}\text { S100A9 } \\
\leq 242.5 \\
87\end{array}$ & $\begin{array}{l}\text { S100A9 } \\
>242.5 \\
69\end{array}$ & $\begin{array}{l}P \\
0.990\end{array}$ \\
\hline & Female & 12 & 10 & & 19 & 15 & \\
\hline \multirow[t]{2}{*}{ Age } & $\leq 50$ & 25 & 34 & 0.144 & 32 & 32 & 0.252 \\
\hline & $>50$ & 70 & 60 & & 74 & 52 & \\
\hline \multirow{2}{*}{$\begin{array}{l}\text { AFP } \\
(\mathrm{ng} / \mathrm{mL})\end{array}$} & $\leq 400$ & 72 & 62 & 0.137 & 68 & 47 & 0.251 \\
\hline & $>400$ & 23 & 32 & & 38 & 37 & \\
\hline \multirow{2}{*}{$\begin{array}{l}\text { ALT } \\
(\mathrm{U} / \mathrm{L})\end{array}$} & $\leq 75$ & 87 & 84 & 0.604 & 92 & 70 & 0.504 \\
\hline & $>75$ & 8 & 10 & & 14 & 14 & \\
\hline \multirow{2}{*}{$\begin{array}{l}\text { Liver } \\
\text { cirrhosis }\end{array}$} & No & 23 & 14 & 0.107 & 15 & 12 & 0.979 \\
\hline & Yes & 72 & 80 & & 91 & 72 & \\
\hline \multirow[t]{2}{*}{ No.of tumor } & Single & 79 & 67 & 0.051 & 93 & 62 & 0.014 \\
\hline & Multiple & 16 & 27 & & 13 & 22 & \\
\hline \multirow{2}{*}{$\begin{array}{l}\text { Tumor size, } \\
(\mathrm{cm})\end{array}$} & $\leq 5$ & 63 & 48 & 0.033 & 69 & 50 & 0.431 \\
\hline & $>5$ & 32 & 46 & & 37 & 34 & \\
\hline \multirow{2}{*}{$\begin{array}{l}\text { Vascular } \\
\text { invasion }\end{array}$} & No & 62 & 57 & 0.510 & 77 & 38 & 0.001 \\
\hline & Yes & 33 & 37 & & 29 & 46 & \\
\hline \multirow{2}{*}{$\begin{array}{l}\text { Edmondson } \\
\text { stage }\end{array}$} & I-II & 62 & 53 & 0.211 & 78 & 36 & 0.001 \\
\hline & III-IV & 33 & 41 & & 28 & 48 & \\
\hline \multirow{2}{*}{$\begin{array}{l}\text { Child-Pugh } \\
\text { score }\end{array}$} & A & 94 & 90 & 0.170 & 100 & 80 & 0.783 \\
\hline & B & 1 & 4 & & 6 & 4 & \\
\hline \multirow[t]{2}{*}{ BCLC stage } & $0+A$ & 82 & 75 & 0.231 & 92 & 63 & 0.037 \\
\hline & $B+C$ & 13 & 19 & & 14 & 21 & \\
\hline
\end{tabular}

Abbreviations: AFP, a-fetoprotein; ALT, alanine aminotransferase; BCLC, Barcelona Clinic Liver Cancer 

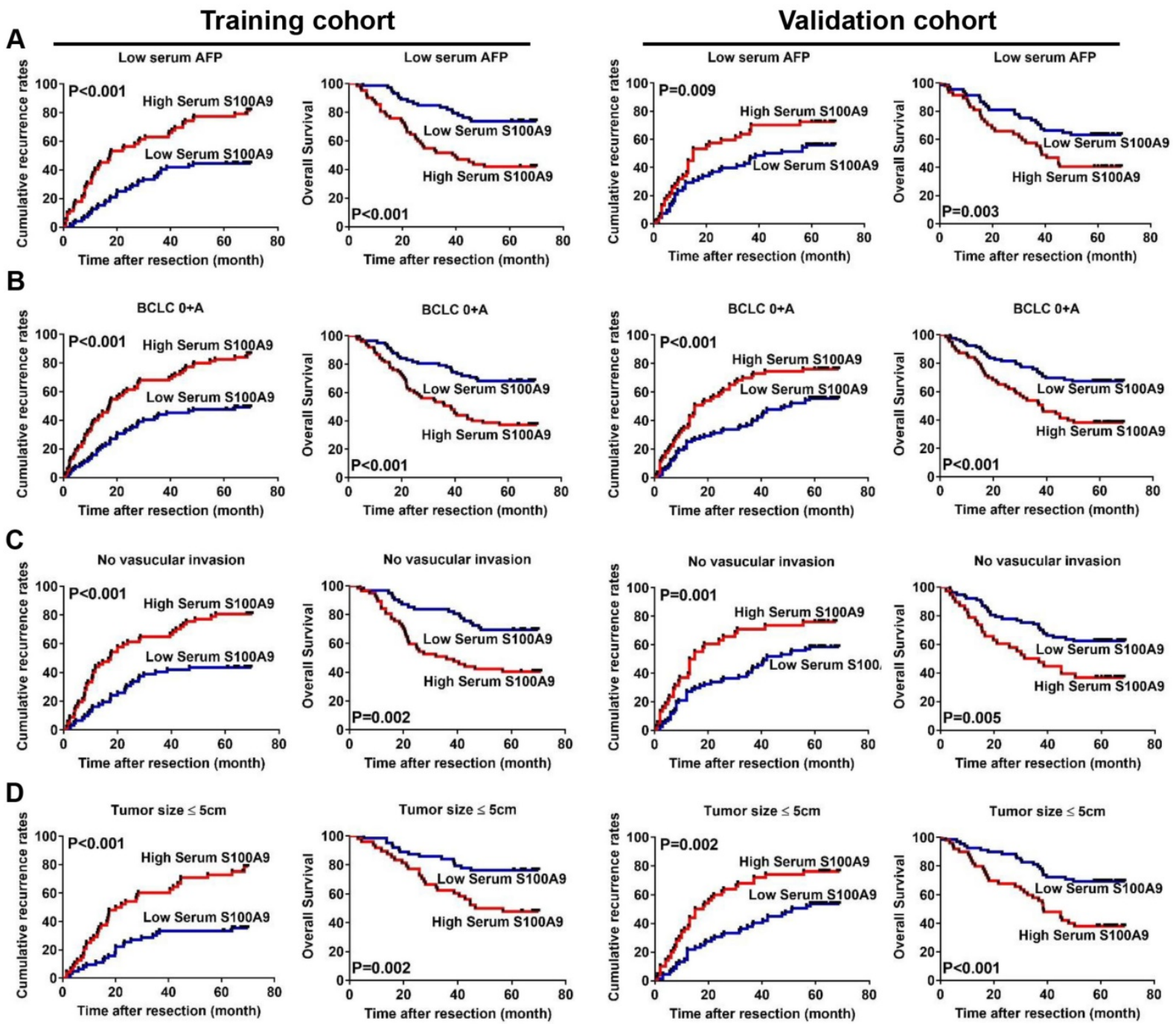

Figure 4. Serum S100A9 retained its prognostic value in low-recurrence-risk subgroups. (A) Kaplan-Meier analysis of $H C C$ patients with low-AFP ( $\leq 400$ ng/ml) according to serum S100A9 levels in training (left two graphs) and validation (right two graphs) cohort. (B) Kaplan-Meier analysis of HCC patients with early-HCC (BCLC $0+A)$ according to serum S100A9 levels in training (left two graphs) and validation (right two graphs) cohort. (C) Kaplan-Meier analysis of HCC patients without vascular invasion according to serum S100A9 levels in training (left two graphs) and validation (right two graphs) cohort. (D) Kaplan-Meier analysis of HCC patients with small-HCC ( $\leq 5 \mathrm{~cm}$ ) according to serum S100A9 levels in training (left two graphs) and validation (right two graphs) cohort.

\section{Discussion}

Determining HCC patients with high risk of developing recurrence remains a dilemma in current HCC surveillance, and few adjuvant treatment strategies are available for patients to help improve OS [25]. In the present study, we demonstrated the clinical significance of serum S100A9 in HCC. Our findings revealed that serum S100A9 was significantly elevated in HCC patients with dismal outcomes. Moreover, serum S100A9 was an independent predictor of both recurrence and death for HCC patients after curative resection, which was validated by an independent cohort of patients. Importantly, S100A9 retained its prognostic value in several conventional low-recurrence/death-risk subgroups, thereby strengthening the clinical utility of this circulating biomarker for predicting HCC prognosis.
Generally, recurrence of HCC is closely correlated with the invasiveness of tumor cells [26-28]. Tumor cells with high invasiveness potential could enter into the circulation, even in early-stage tumors, and spread to non-tumor liver tissues. These cells would then transform into quiescent conditions and would be susceptible to being triggered to develop recurrent lesions after curative resection [24]. S100A9 is considered to be one of the key regulators that contributes to tumor progression via triggering pro-tumor immune responses. Previous studies showed that stromal cells could secrete S100A9 to recruit myeloid cells to the tumor microenvironment, therefore resulting in an immunosuppressive status within the tumor microenvironment to promote tumor progression. Intriguingly, S100A9 was also reported as overexpressed in tumor cells, and tumor-derived S100A9 could function as a contributor 
for tumor migration and invasion, which inevitably leads to the metastasis of tumor cells $[29,30]$.

S100A9 was also proven as a critical contributor for HCC proliferation as well as invasion by activating ERK signaling, and in situ expression of S100A9 significantly correlated with poor prognosis of HCC patients [20]. Because S100A9 is a secreted protein, detection of serum S100A9 concentration might be a promising approach to distinguish patients with high risk of recurrence. Our data were well in line with previous findings showing that serum S100A9 was upregulated in HCC and patients with high preoperative S100A9 level showed significantly shorter TTR and OS [21]. Importantly, S100A9 level served as an independent indicator for dismal outcomes of HCC patients. Determination of S100A9 involves a serum-based methodology, which exhibits advantages in easy handling, cost effectiveness, and high reproducibility. Our results indicate S100A9 is a novel and reliable parameter for predicting prognosis after resection. Patients with high serum S100A9 should be paid more attention and more precise imaging scans should be applied to detect early recurrence. Moreover, adjuvant treatment might be applied to these patients to prevent recurrence.

In clinical practices, the prediction of individuals that will show poor outcome after curative resection for early-stage HCC has remained difficult [31-33]. We found that serum S100A9 level retained its clinical significance in BCLC 0+A-stage patients with HCC. Currently, AFP is the most widely used serum biomarker for HCC. However, approximately $30 \%$ to $40 \%$ of patients have normal serum AFP levels at diagnosis, which is a significant limitation for the clinical use of this biomarker. We investigated the prognostic value of S100A9 in the low-AFP subgroup and found that low-AFP patients could be stratified into two groups according to serum S100A9 with distinct outcomes. Our results suggested that S100A9 is a powerful prognostic marker for HCC, especially for patients with early-stage disease and normal AFP levels. These results indicate serum S100A9 as a novel serum biomarker for patients whose prognosis is difficult to predict by conventional indices.

There were several limitations in the present study. Most of our enrolled patients had HBV background, which means that the clinical significance needs to be further validated in patients with other etiology background [34]. In addition, the origin of high serum S100A9 also needs to be comprehensively investigated by histopathology assays. However, it should be noted that we constructed an independent cohort of patients to validate the clinical utility of serum S100A9, and the clinical characteristics between the training and validation cohorts were well balanced, which indicates the reliability and universality of our findings.

\section{Conclusions}

To the best of our knowledge, this is the first report to demonstrate the prognostic prediction value of serum S100A9 in HCC patients. We confirmed that determination of serum S100A9 level might provide useful information to facilitate the implementation of different treatment approaches. Because the quantification of S100A9 in circulation is easy and reproducible using ELISA, it lends itself to wide applicability. Further investigation into the origin of S100A9 and its role in regulating the tumor microenvironment might provide a new insight into the mechanism of HCC recurrence and metastasis. This information may identify novel therapeutic strategies that target both the tumor cell itself and microenvironment to improve the prognosis of HCC patients.

\section{Abbreviations}

HCC, hepatocellular carcinoma; BCLC, Barcelona Clinic Liver Cancer; TTR, time to recurrence; OS, overall survival; ELISA, enzyme-linked immunosorbent assay; HBV, hepatitis B virus; AFP, alphafetoprotein; HR, hazard ratio; 95\% CI, 95\% confidential interval.

\section{Competing Interests}

The authors have declared that no competing interest exists.

\section{References}

[1] Nault JC, Galle PR, Marquardt JU. The role of molecular enrichment on future therapies in hepatocellular carcinoma. J Hepatol. 2018; 69: 237-247.

[2] Ringelhan M, Pfister D, O'Connor T, et al. The immunology of hepatocellular carcinoma. Nat Immunol. 2018; 19: 222-232

[3] Wong CM, Tsang FH, Ng IO. Non-coding RNAs in hepatocellular carcinoma: molecular functions and pathological implications. Nat Rev Gastroenterol Hepatol. 2018; 15: 137-151

[4] Inarrairaegui M, Melero I, Sangro B. Immunotherapy of Hepatocellular Carcinoma: Facts and Hopes. Clin Cancer Res. 2018; 24: 1518-1524

[5] Greten TF, Sangro B. Targets for immunotherapy of liver cancer. J Hepatol 2017. (17)32287-0 [Epub ahead of print].

[6] Finn RS, Zhu AX, Farah W, et al. Therapies for advanced stage hepatocellular carcinoma with macrovascular invasion or metastatic disease: A systematic review and meta-analysis. Hepatology. 2018; 67: 422-435.

[7] Roberts LR, Sirlin CB, Zaiem F, et al. Imaging for the diagnosis of hepatocellular carcinoma: A systematic review and meta-analysis. Hepatology. 2018; 67: 401-421.

[8] Bouattour M, Raymond E, Qin S, et al. Recent developments of c-Met as a therapeutic target in hepatocellular carcinoma. Hepatology. 2018; 67: 1132-1149.

[9] Wen H, Ma H, Cai Q, et al. Recurrent ECSIT mutation encoding V140A triggers hyperinflammation and promotes hemophagocytic syndrome in extranodal NK/T cell lymphoma. Nat Med. 2018; 24: 154-164.

[10] Goh JY, Feng M, Wang W, et al. Chromosome 1q21.3 amplification is a trackable biomarker and actionable target for breast cancer recurrence. Nat Med. 2017; 23: 1319-1330.

[11] Laouedj M, Tardif MR, Gil L, et al. S100A9 induces differentiation of acute myeloid leukemia cells through TLR4. Blood. 2017; 129: 1980-1990. 
[12] Hansen MT, Forst B, Cremers N, et al. A link between inflammation and metastasis: serum amyloid $\mathrm{A} 1$ and $\mathrm{A} 3$ induce metastasis, and are targets of metastasis-inducing S100A4. Oncogene. 2015; 34: 424-35.

[13] Tidehag V, Hammarsten P, Egevad L, et al. High density of S100A9 positive inflammatory cells in prostate cancer stroma is associated with poor outcome. Eur J Cancer. 2014; 50: 1829-35.

[14] Eisenblaetter M, Flores-Borja F, Lee JJ, et al. Visualization of Tumor-Immune Interaction - Target-Specific Imaging of S100A8/A9 Reveals Pre-Metastatic Niche Establishment. Theranostics. 2017; 7: 2392-2401

[15] Gebhardt C, Nemeth J, Angel P, et al. S100A8 and S100A9 in inflammation and cancer. Biochem Pharmacol. 2006; 72: 1622-31

[16] Gebhardt C, Riehl A, Durchdewald M, et al. RAGE signaling sustains inflammation and promotes tumor development. J Exp Med. 2008; 205: 275-85

[17] Kim WT, Kim J, Yan C, et al. S100A9 and EGFR gene signatures predict disease progression in muscle invasive bladder cancer patients after chemotherapy. Ann Oncol. 2014; 25: 974-9

[18] Nemeth J, Stein I, Haag D, et al. S100A8 and S100A9 are novel nuclear factor kappa B target genes during malignant progression of murine and human liver carcinogenesis. Hepatology. 2009; 50: 1251-62

[19] Wilson CL, Jurk D, Fullard N, et al. NFkappaB1 is a suppressor of neutrophil-driven hepatocellular carcinoma. Nat Commun. 2015; 6: 6818

[20] Wu R, Duan L, Cui F, et al. S100A9 promotes human hepatocellular carcinoma cell growth and invasion through RAGE-mediated ERK1/2 and p38 MAPK pathways. Exp Cell Res. 2015; 334: 228-38.

[21] Sun W, Xing B, Guo L, et al. Quantitative Proteomics Analysis of Tissue Interstitial Fluid for Identification of Novel Serum Candidate Diagnostic Marker for Hepatocellular Carcinoma. Sci Rep. 2016; 6: 26499

[22] Bruix J, Sherman M. Management of hepatocellular carcinoma: an update. Hepatology. 2011; 53: 1020-2

[23] Ma XL, Zhou JY, Gao XH, et al. Application of the albumin-bilirubin grade for predicting prognosis after curative resection of patients with early-stage hepatocellular carcinoma. Clin Chim Acta. 2016; 462: 15-22

[24] Wu J, Ma XL, Tian L, et al. Serum IgG4:IgG Ratio Predicts Recurrence of Patients with Hepatocellular Carcinoma after Curative Resection. J Cancer. 2017; 8: 1338-1346

[25] Yen CJ, Ai YL, Tsai HW, et al. Hepatitis B virus surface gene pre-S2 mutant as a high-risk serum marker for hepatoma recurrence after curative hepatic resection. Hepatology 2018; doi: 10.1002/hep.29790 [Epub ahead of print].

[26] Guo W, Sun YF, Shen MN, et al. Circulating Tumor Cells with Stem-Like Phenotypes for Diagnosis, Prognosis, and Therapeutic Response Evaluation in Hepatocellular Carcinoma. Clin Cancer Res. 2018; 24: 2203-2213.

[27] Ma XL, Gao XH, Gong ZJ, et al. Apolipoprotein A1: a novel serum biomarker for predicting the prognosis of hepatocellular carcinoma after curative resection. Oncotarget. 2016; 7: 70654-70668

[28] Lu M, Wu J, Hao ZW, et al. Basolateral CD147 induces hepatocyte polarity loss by E-cadherin ubiquitination and degradation in hepatocellular carcinoma progress. Hepatology. 2018; 68: 317-332.

[29] Yang $\mathrm{O}, \mathrm{Li} \mathrm{X}$, Chen $\mathrm{H}$, et al. IRF7 regulates the development of granulocytic myeloid-derived suppressor cells through S100A9 transrepression in cancer. Oncogene. 2017; 36: 2969-2980

[30] $\mathrm{Pu} \mathrm{W}$, Li J, Zheng Y, et al. Targeting Pin1 by inhibitor API-1 regulates microRNA biogenesis and suppresses hepatocellular carcinoma development. Hepatology. 2018; 68: 547-560.

[31] Guo W, Yang XR, Sun YF, et al. Clinical significance of EpCAM mRNA-positive circulating tumor cells in hepatocellular carcinoma by an optimized negative enrichment and qRT-PCR-based platform. Clin Cancer Res. 2014; 20: 4794-805

[32] Kadalayil L, Benini R, Pallan L, et al. A simple prognostic scoring system for patients receiving transarterial embolisation for hepatocellular cancer. Ann Oncol. 2013; 24: 2565-70

[33] Kurebayashi $\mathrm{Y}$, Ojima $\mathrm{H}$, Tsujikawa $\mathrm{H}$, et al. Landscape of immune microenvironment in hepatocellular carcinoma and its additional impact on histological and molecular classification. Hepatology 2018; doi:10.1002/hep.29904 [Epub ahead of print].

[34] Hu B, Yang XR, Xu Y, et al. Systemic immune-inflammation index predicts prognosis of patients after curative resection for hepatocellular carcinoma. Clin Cancer Res. 2014; 20: 6212-22 